\title{
CLASSIFICATION OF CONTINUOUS FLOWS ON 2-MANIFOLDS
}

\author{
DEAN A. NEUMANN
}

ABSTRACT. We prove that a continuous flow with isolated critical points on an arbitrary 2 -manifold is determined up to topological equivalence by its separatrix configuration.

1. Introduction. In [3] Markus proves the following result: If $\phi$ is a $C^{1}$ flow on the plane, with isolated critical points and no limit separatrices other than critical points, then $\phi$ is determined up to topological equivalence by its separatrix configuration. The purpose of the present paper is to extend this result to continuous flows on arbitrary 2-manifolds and remove the restriction on limit separatrices.

2. Definitions and preliminaries. Let $M$ denote a 2-manifold (separable metric, connected and without boundary, but not necessarily compact nor orientable) and $\phi: M \times \mathbf{R}^{1} \rightarrow M$ a continuous flow on $M$. Two such flows, $\left(M_{1}, \phi_{1}\right)$ and $\left(M_{2}, \phi_{2}\right)$, are (topologically) equivalent if there is a homeomorphism of $M_{1}$ onto $M_{2}$ which takes orbits of $\phi_{1}$ onto orbits of $\phi_{2}$, preserving sense.

We call $(M, \phi)$ parallel if it is equivalent to one of the following:

1. $\mathbf{R}^{2}$ with flow defined by $y^{\prime}=0$;

2. $\mathbf{R}^{2}-\{0\}$ with flow defined (in polar coordinates) by $d r / d t=0$, $d \theta / d t=1$

3. $\mathbf{R}^{2}-\{0\}$ with flow defined by $d r / d t=r, d \theta / d t=0$;

4. $S^{1} \times S^{1}$ with 'rational' flow (e.g., the flow induced by (1) above, under the usual covering map).

We distinguish these as strip, annular, spiral (or radial) and toral respectively.

Throughout this paper we consider flows $(M, \phi)$ with isolated critical points. Denote the orbit ( \pm semiorbit) of $x \in M$ by $\gamma(x)\left(\gamma^{ \pm}(x)\right)$ and let

$$
\alpha(x)=\overline{\gamma^{-(x)}}-\gamma^{-(x),} \quad \omega(x)=\overline{\gamma^{+(x)}}-y^{+}(x) \text {. }
$$

Received by the editors December 12, 1973.

AMS (MOS) subject classifications (1970). Primary 34C35; Secondary 34C40.

Key words and phrases. Flows on 2-manifolds, separatrices. 
We say that $\gamma(x)$ is a separatrix of $\phi$ (cf. [3]) if $\gamma(x)$ is not contained in a parallel neighborhood $N$ satisfying both:

1. for all $y \in N, a(y)=a(x)$ and $\omega(y)=\omega(x)$;

2. $\bar{N}-N$ consists of $\alpha(x), \omega(x)$ and exactly two orbits $\gamma(a), \gamma(b)$ of $\phi$ with $\alpha(a)=\alpha(b)=\alpha(x)$ and $\omega(a)=\omega(b)=\omega(x)$.

Let $S$ denote the union of all separatrices of $\phi$-so $S$ is a closed invariant subset of $M$. A component of the complement, with the restricted flow, is called a canonical region of $\phi$.

Lemma. Any canonical region of $(M, \phi)$ is parallel.

Proof. Let $\left(R, \phi^{\prime}=\phi \mid R\right)$ be a canonical region. There are no separatrices in $R$, so the set consisting of orbits homeomorphic with $S^{1}$ is open, and similarly for the set consisting of line homeomorphs. Hence $R$ consists entirely of closed orbits or entirely of line orbits.

Also, two orbits of $\phi^{\prime}$ can be separated with disjoint parallel neighborhoods. For suppose $\gamma(x)$ and $\gamma(y)$ are distinct orbits (closed or not) which cannot be separated. Then, for any parallel neighborhood $N_{x}$ of $x$, we have $y \in \bar{N}_{x}$; i.e., $y \in \bigcap \bar{N}_{x}=\alpha(x) \cup \gamma(x) \cup \omega(x)$. But then $y \in \alpha(x)$ (or $y \in \omega(x)$ ) and this is impossible since $y$ lies in a parallel neighborhood which may be taken to exclude $\gamma(x)$.

It follows that the quotient space $R / \phi^{\prime}$ is a (Hausdorff) 1 -manifold and hence that the natural projection $\pi: R \rightarrow R / \phi^{\prime}$ is a locally trivial fibering of $R$ over $\mathbf{R}^{1}$ or $S^{1}$, with fibers homeomorphic to $\mathbf{R}^{1}$ or $S^{1}$. Since the flow provides a natural orientation on the fibers, there are only four possibilities-the four classes of parallel flows described above.

A separatrix configuration for $(M, \phi)$, denoted $S^{+}$, is the union of all separatrices of $\phi$ together with a representative orbit from each canonical region of $\phi$. Separatrix configurations, $S_{1}^{+}$for $\left(M_{1}, \phi_{1}\right)$ and $S_{2}^{+}$for $\left(M_{2}, \phi_{2}\right)$ are equivalent if there is a homeomorphism of $M_{1}$ onto $M_{2}$ taking orbits of $\left(S_{1}^{+}, S_{1}\right)$ onto those of $\left(S_{2}^{+}, S_{2}\right)$, preserving sense. A separatrix $\gamma(x)$ of $\phi$ is called a limit separatrix if $\gamma(x)$ is in the closure of $S-\gamma(x)$.

It follows from the Lemma that any canonical region $R$ admits a complete transversal; i.e., a section which meets each orbit of $R$ exactly once. We will also use repeatedly the fact that through any nonrest point of $(M, \phi)$ there is a local section $S$, with $S[-\epsilon, \epsilon]$ homeomorphic to the rectangle $\{(s, t)|s \in[-1,1]| t \mid, \leq \epsilon\}$ under the map $(s, t) \rightarrow \phi(\alpha(s), t)=\alpha(s) \cdot t$, where $\alpha:[-1,1] \rightarrow S$ defines the section $S$. If $x$ is wandering, we may take $\epsilon=\infty$ (see [1, Chapter IV, $\$ 2]$, and [2, Theorem 1]).

If $R$ is a canonical region of $(M, \phi)$, let $\partial R$ denote $\bar{R}-R$. In the 
simplest situations each noncritical point of $\partial R$ is accessible from $R$ as the endpoint of a (local) section of $\phi$ which otherwise lies in $\dot{R}$. However, there may be limit separatrices in $\partial R$ which are not accessible from $R$ (for example, if we insert a rest point into a spiral region with a limit cycle, we obtain a strip region $R$, with the limit cycle in $\partial R$ but not accessible from $R)$. Hence we distinguish the union of separatrices accessible from $R$ as the accessible boundary of $R$, and denote it by $\delta R$. It is not hard to show that every (noncritical) boundary point of a spiral or annular region $R$ is accessible from $R$.

Finally, we distinguish two types of spiral regions. Suppose $\gamma(m)$ is an orbit of the spiral region $R$ and that both $\alpha(m)$ and $\omega(m)$ contain noncritical points. We say that

1. $R$ is orientable if the orientations on separatrices of $\delta R$ induced by the flow are compatible with some orientation of $R$ (cf. Figure 1);

2. $R$ is nonorientable otherwise.

We say that an arc spans a canonical region $R$, if it is a (local) section which lies in $R$ except for its endpoints. Note that there can be no spanning section in an orientable spiral region. Hence such regions cannot accumulate at a noncritical limit separatrix.

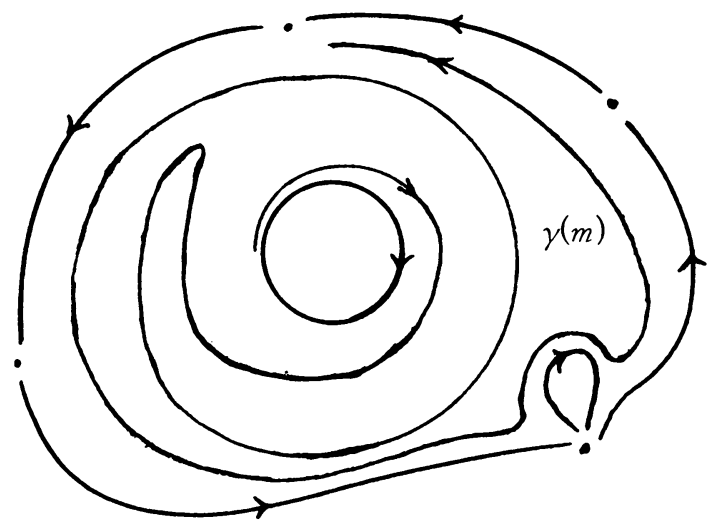

Figure 1

3. Subdivisions of canonical regions. Suppose $\phi_{1}$ and $\phi_{2}$ are continuous flows on $M$ with isolated critical points and the same separatrix configuration $S^{+}$. For each canonical region $R$, we wish to describe an equivalence $h$ of $\left(R, \phi_{1}\right)$ with $\left(R, \phi_{2}\right)$ which extends by the identity to an equivalence on $R \cup \delta R$. We do this by constructing subdivisions of $\left(R, \phi_{1}\right)$, $\left(R, \phi_{2}\right)$ which 'converge' at $\delta R$, and defining $h$ to be 'cellular' in these subdivisions. The construction also restricts $h$ on the interior of $R$ in 
such a way that the composite equivalence (obtained by piecing together the various canonical regions) extends continuously to the limit separatrices. The latter restriction is measured by a positive constant $\epsilon$, which we assume fixed for the remainder of this section.

It is convenient to pass to the manifold $\breve{M}$ consisting of $M$ minus the critical points of $\phi_{i}$; we denote the restricted flows by $\phi_{i}$ also. We may assume that the topology of $\check{M}$ is defined by a complete metric $\rho$. The constructions of this section refer to $\left(\check{M}, \phi_{i}\right)$.

Strip canonical regions. Let $R$ be a strip region and let $\gamma(m) \subset S^{+}$be the distinguished orbit. Choose points $p_{k} \in \gamma(m)(k \in \mathbf{Z})$ satisfying:

1. $p_{k}=m t_{k}$ where $t_{k}$ strictly increases with $k$ and is unbounded above and below;

2. $\rho\left(p_{k}, p_{k+1}\right)<\epsilon(k \in \mathbf{Z})$;

3. if $\alpha(m) \neq \varnothing(\omega(m) \neq \varnothing)$ then $\lim _{k \rightarrow-\infty} \rho\left(p_{k}, p_{k+1}\right)=0$ $\left(\lim _{k \rightarrow \infty} \rho\left(p_{k}, p_{k+1}\right)=0\right)$.

Note that $\gamma(m)$ separates $R$ into two half-regions $R^{+}$and $R^{-}$(both containing $\gamma(m)$ ). If $\delta R \neq \varnothing$, we construct a subdivision of $R^{+} ; R^{-}$is treated similarly.

Define

$$
a_{k}=\inf \left\{a>0 \mid \exists \text { a section of } \phi_{1} \text { from } p_{k} \text { to } \delta R^{+} \text {of diameter } a\right\} .
$$

Let $A \subset \mathbf{Z}$ consist of 0 and those indices $k$ for which $a_{k} \leq 1$. Construct disjoint sections $S_{k}(k \in A)$ of $\phi_{1}$ from $p_{k}$ to points $q_{k} \in \delta R^{+}$, with $\operatorname{diam}\left(S_{k}\right)<2 a_{k}$. We may see that this is possible as follows. If we have already constructed $n$ such sections we can add another, possibly having to adjust some of the previously constructed ones to insure disjointness. However, the section at a given $p_{k_{0}}$ need be adjusted only a finite number of times in this process. If $\omega(m) \stackrel{0}{=} \varnothing(\alpha(m)=\varnothing)$, this follows from the fact that $\rho\left(p_{k}, p_{k}\right) \rightarrow \infty$ as $k \rightarrow \infty(k \rightarrow-\infty)$ (because $\rho$ is complete), while the sections constructed have bounded diameters. If $\omega(m) \neq \varnothing(\alpha(m) \neq \varnothing)$, then there are indices for which $a_{k}$ is arbitrarily small; once $S_{k_{0}}$ is adjusted to miss a sufficiently small section, subsequent sections may be

chosen disjoint from $S_{k_{0}}$ without altering it.
Next, for $k \in A$, let

$$
b_{k}=\inf \left\{b>0 \mid \exists \text { a section of } \phi_{2} \text { from } p_{k} \text { to } q_{k} \text { of diameter } b\right\} \text {, }
$$

and construct disjoint sections $S_{k}^{\prime}$ of $\phi_{2}$ from $p_{k}$ to $q_{k}$ with $\operatorname{diam}\left(S_{k}^{\prime}\right)<2 b_{k}$.

Finally, let $\left\{d_{k}\right\}_{k \in Z^{+}}$be a countable dense subset of the separatrices of $\delta R^{+}$which is disjoint from $\left\{q_{k}\right\}_{k \in A}$. Construct disjoint sections $T_{k}$ of 
$\phi_{1}$ and $T_{k}^{\prime}$ of $\phi_{2}$, both terminating at $d_{k}$ and satisfying (cf. Figure 2):

1. $T_{k}$ is disjoint from every $S_{k}\left(T_{k}^{\prime}\right.$ is disjoint from every $\left.S_{k}^{\prime}\right)$;

2. $\operatorname{diam}\left(T_{k}\right) \rightarrow 0\left(\operatorname{diam}\left(T_{k}^{\prime}\right) \rightarrow 0\right)$ as $k \rightarrow \infty$;

3. if $T_{k}\left(T_{k}^{\prime}\right)$ has initial point on the orbit $\gamma_{1}\left(r_{k}\right)$ of $\phi_{1}\left(\gamma_{2}\left(r_{k}^{\prime}\right)\right.$ of $\left.\phi_{2}\right)$, where $r_{k} \in S_{0}\left(r_{k}^{\prime} \in S_{0}^{\prime}\right)$, then $r_{k}\left(r_{k}^{\prime}\right)$ converges monotonically to $q_{0}$.

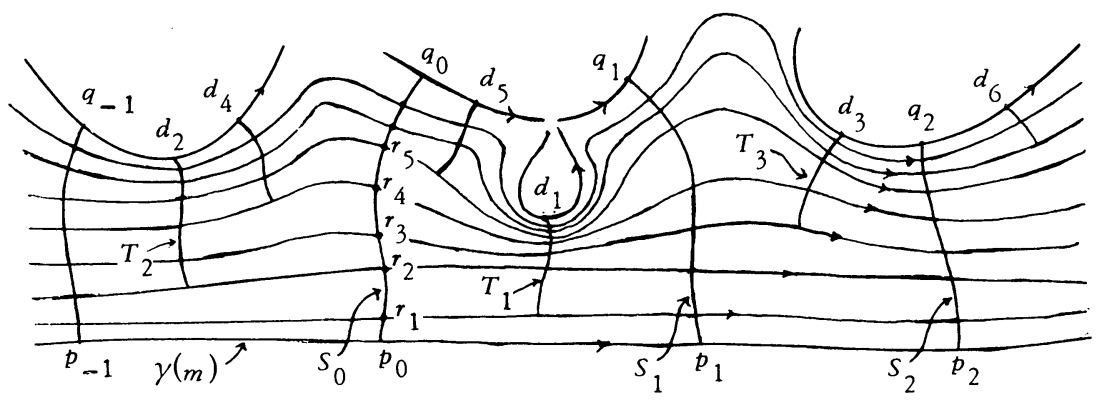

Figure 2

The sections $S_{k}, T_{k}$ and the orbits $\gamma_{1}\left(r_{k}\right)$ then partition $R^{+}$into a locally-finite collection of 2 -cells (those at the 'ends' of $R^{+}$missing a closed subarc of their boundaries), which we refer to as an $\epsilon$-subdivision of $R^{+}$with respect to $\phi_{1}$. The $S_{k}^{\prime}, T_{k}^{\prime}$ and $\gamma_{2}\left(r_{k}^{\prime}\right)$ provide an 'isomorphic' subdivision of $R^{+}$with respect to $\phi_{2}$. It follows that there is an equivalence $h$ of $\left(R^{+}, \phi_{1}\right)$ onto $\left(R^{+}, \phi_{2}\right)$ which takes cells of one subdivision onto the corresponding cells of the other. If we define $h$ to be the identity on $\delta R^{+}$ then the extended function is continuous at $\delta R^{+}$. If $p \in \delta R^{+}$is not in $\alpha(m) \cup \omega(m)$, this follows easily from the construction: let $U$ be an arbitrary neighborhood of $p$ in $R^{+} \cup \delta R^{+}$; pick $i, j$ and $l$ so that the neighborhoods $N\left(N^{\prime}\right)$ of $p$ bounded by segments of $T_{i}, T_{j} T_{j}$ and $\gamma_{1}\left(r_{l}\right)\left(T_{i}^{\prime}, T_{j}^{\prime}\right.$ and $\left.\gamma_{2}\left(r_{l}^{\prime}\right)\right)$ both lie in $U$; then $h(N)=N^{0} \subset U$. If $p \in a(m) \cup \omega(m)$, then $\gamma(p)$ is a limit separatrix and continuity of $h$ at $p$ follows by the general argument for limit separatrices given in $\$ 4$.

If $\delta R^{+}=\varnothing$ then $\partial R=\varnothing$ and we may take $h$ to be any equivalence of $\left(R, \phi_{1}\right)$ with $\left(R, \phi_{2}\right)$ which is the identity on $\gamma(m)$.

Annular canonical regions. Here the construction is exactly as above, except that $\left\{p_{k}\right\}$ is now a finite sequence, spaced less than $\epsilon$ apart and monotonic along the distinguished orbit $\gamma(m)$.

Spiral canonical regions. First suppose that $R$ is a nonorientable spiral region. Let $S_{0}$ be a local section of $\phi_{1}$ which spans $R$ and has diameter less than twice the infimum for such sections. Let $p$ and $q$ be two successive intersections of $\gamma^{+}(m)$ with $S_{0}$ and pick $p_{0}=p, p_{1}, \cdots, p_{n}, p_{n+1}=$ $q$ monotonic along $\gamma^{+}\left(p_{0}\right)$ and spaced closer together than $\epsilon$. Let $C$ denote 
the simple closed curve consisting of $\left[p_{0}, p_{n+1}\right] \subset \gamma^{+}(m)$ and $\left[p_{0}, p_{n+1}\right] \subset$ $S_{0}$, and define $R^{+}=C[0, \infty), R^{-}=C(-\infty, 0]$.

Construct disjoint sections $S_{k}$ of $\phi_{1}$ from $p_{k}$ to points $q_{k} \in \delta R^{+}$of diameter less than twice the infimum of possible diameters. Then construct sections $S_{k}^{\prime}$ to $\phi_{2}$ from $p_{k}$ to $q_{k}$ with the analogous restriction on diameters.

If $R$ is orientable (or if $\alpha(m)$ or $\omega(m)$ is empty), then we need not construct such spanning sections. However, we may define the analogues of $C, R^{+}$and $R^{-}$in these cases also.

Finally, for any spiral region $R$ with $\delta R^{+} \neq \varnothing$, let $\left\{d_{k}\right\}(k \geq 1)$ be a countable dense subset of $\delta R^{+}$(disjoint from $\left\{q_{k}\right\}$ in the nonorientable case) and construct local sections $T_{k}\left(T_{k}^{\prime}\right)$ to $\phi_{1}\left(\phi_{2}\right.$ respectively) satisfying (cf. Figure 3):

1. $T_{k}$ and $T_{k}^{\prime}$ originate at the same point of $\gamma(m)$;

2. $T_{k}$ and $T_{k}^{\prime}$ terminate at $d_{k}$;

3. $\operatorname{diam}\left(T_{k}\right) \rightarrow 0$ and $\operatorname{diam}\left(T_{k}^{\prime}\right) \rightarrow 0$.

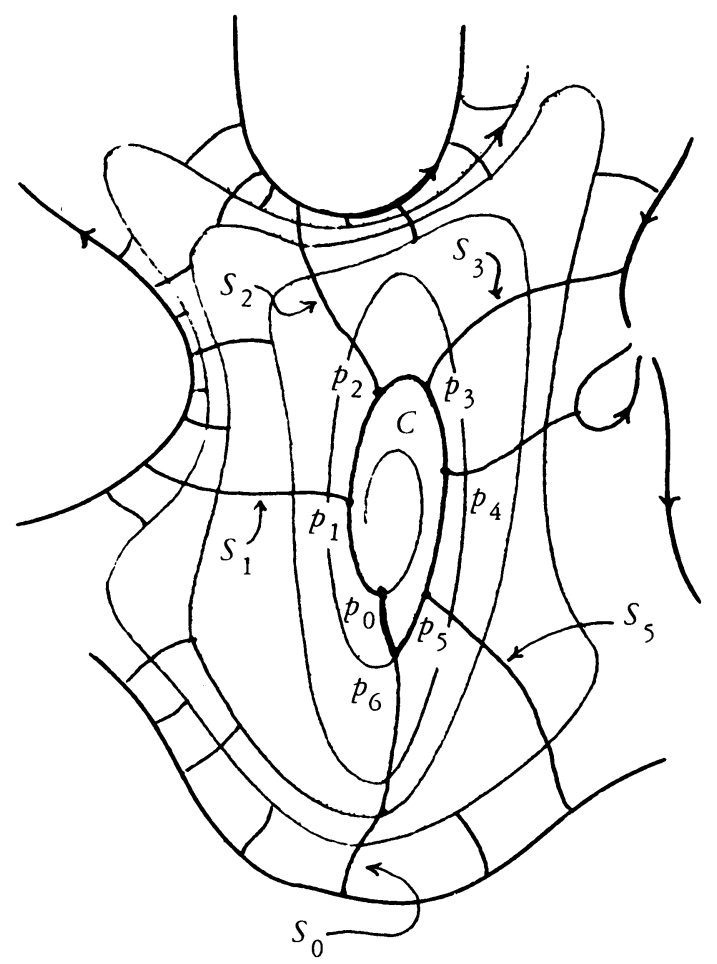

Figure 3 
There is an equivalence $h$ of $\left(R^{+}, \phi_{1}\right)$ onto $\left(R^{+}, \phi_{2}\right)$ which is the identity on $C$, and takes cells of one partition onto corresponding cells of the other. As in the case of strip regions, such an equivalence extends continuously to $\delta R^{+}$by the identity.

If $\delta R^{+}=\varnothing$, take $h$ to be any equivalence which is the identity on $C$. $R^{-}$is treated similarly.

Toral canonical regions. If $R$ is a toral region then $R=M$. Let $h$ be any equivalence of $\left(M, \phi_{1}\right)$ with $\left(M, \phi_{2}\right)$.

\section{Classification theorem.}

Theorem. Suppose $\phi_{1}$ and $\phi_{2}$ are continuous flows on the 2-manifold $M$, with isolated critical points. Then $\phi_{1}$ and $\phi_{2}$ are equivalent iff their separatrix configurations are equivalent.

Proof. (Sufficiency). Suppose $k$ is an equivalence of $\left(S_{1}^{+}, S_{1}\right)$ with $\left(S_{2}^{+}, S_{2}\right)$. If $h$ is a homeomorphism of $M$ which is the identity on $S_{2}$, and an equivalence of the flow induced by $\phi_{1}$ under $k$ with $\phi_{2}$, then $h k$ is the required equivalence. Hence we may assume that $\phi_{1}$ and $\phi_{2}$ have the same separatrix configuration $S^{+}$, and construct $h$.

Order the canonical regions $\left\{R_{n}\right\} n \geq 1$. Let $\gamma\left(m_{n}\right)$ denote the distinguished orbit of $R_{n}$. For each $n$, define $1 / n$-subdivisions of $R_{n}$ with respect to $\phi_{1}$ and $\phi_{2}$, as above. By the results of $\$ 3$, there is a cellular equivalence of $\left(R_{n}, \phi_{1}\right)$ with $\left(R_{n}, \phi_{2}\right)$, which extends by the identity to nonlimit separatrices of $\delta R_{n}$. Define $h$ to be the identity on $S^{+}$; we need to prove that $h$ is continuous at limit separatrices.

First suppose $p$ is a noncritical point on a limit separatrix and fix $\epsilon>0$. Then $\gamma(p)$ separates a neighborhood $U$ of $p$ into two components; at least one of these, say $H$, meets separatrices which accumulate at $p$. 'Let $N$ denote a closed trivial neighborhood of $p$ in $\bar{H}$ which is bounded by local sections of $\phi_{1}$ terminating on $\gamma(p)$, and a segment of a separatrix $\gamma(q)$ (cf. Figure 4), and let $N^{\prime} \subset N$ be similarly bounded by $\gamma(q)$ and sections of $\phi_{2}$. Let $h_{1}\left(h_{2}^{\prime}\right)$ be a homeomorphism of $N\left(N^{\prime}\right)$ onto $D=\{(x, y)|| x \mid \leq$ $1,0 \leq y \leq 1\}$, taking orbit segments of $\phi_{1}\left(\phi_{2}\right)$ onto horizontal segments and taking $p$ to 0 . Let $k: D \rightarrow D$ be an embedding which extends the map $\left.h_{1} \circ h_{2}^{\prime}{ }^{-1}\right|_{h_{2}^{\prime}\left(N^{\prime} \cap S^{+}\right)}$, and maps horizontal segments to horizontal segments. Define $h_{2}: N^{\prime} \rightarrow D$ by $h_{2}=k h_{2}^{\prime}$. Then $h_{2}=b_{1}$ " on $N^{\prime} \cap S^{+}$.

Choose $B>0$ satisfying $B^{-1} \operatorname{diam}(S) \leq \operatorname{diam} h_{i}(S) \leq B \operatorname{diam}(S)$ for any subset $S \subset N^{\prime}$ and $i=1,2$. Pick $a>0$ so that $Q=\{(x, y) \| x \mid \leq a, 0 \leq y \leq 1\}$ 


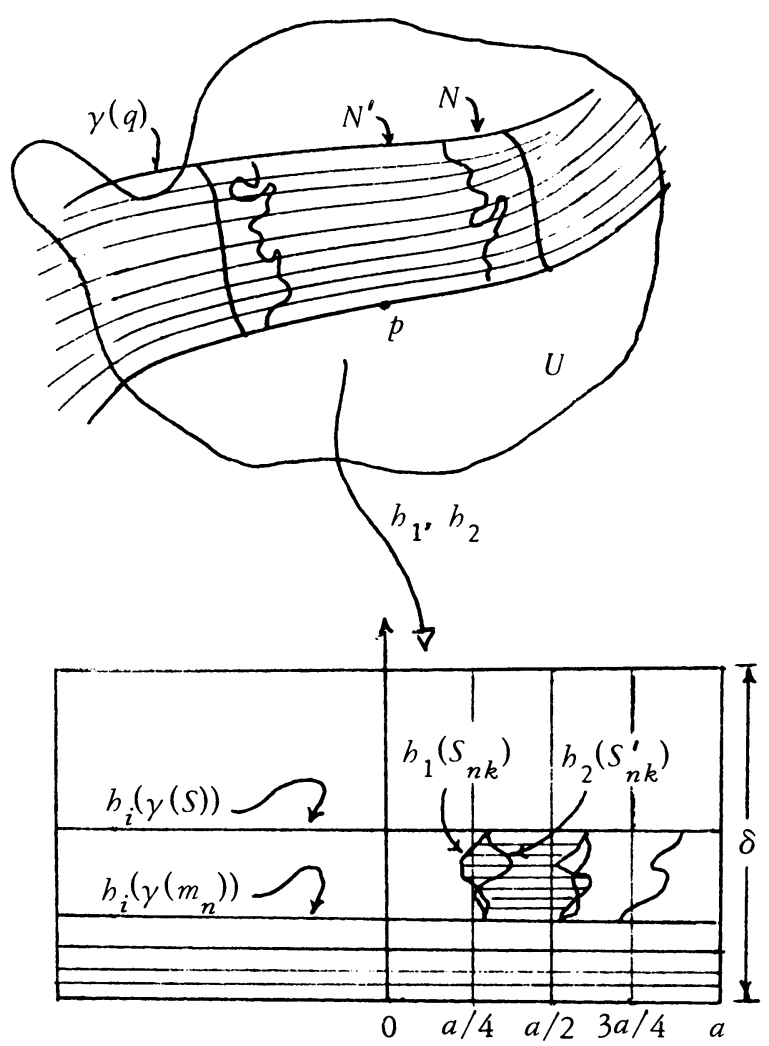

Figure 4

is contained in both $h_{1}\left(N^{\prime}\right), h_{2}\left(N^{\prime}\right)$. Set $m=\min \{\epsilon, a / 4\} . Q \cap h_{i}\left(S^{+}\right)$contains segments arbitrarily close to 0 . The complement of these consists of 'strips' which are the intersections of the images of the various half canonical regions with $Q$.

Now choose $\delta>0$ so that the set $Q_{\delta}=\{(x, y)|| x \mid \leq a, 0 \leq y \leq \delta\}$ satisfies the following:

1. the supremum of widths of strips meeting $Q_{\delta}$ is less than $m / 2 B^{2}$;

2. the spacing between successive $h_{i}\left(p_{n k}\right)$ along the image of any segment of a distinguished orbit $\gamma\left(m_{n}\right)$ which meets $Q_{\delta}$ is less than $m$.

Let $S$ be a strip in $Q_{\delta}$, bounded by segments of $h_{i}\left(\gamma\left(m_{n}\right)\right)$ and $h_{i}(\gamma(s))$ (so $\gamma(s) \subset \delta R$ is a separatrix). Consider any of the points $h_{i}\left(p_{n k}\right)$ lying between $x=-3 a / 4$ and $x=3 a / 4$. There is a section across $S$, of both $h_{1} \phi_{1}$ and $h_{2} \phi_{2}$, with diameter less than $m / 2 B^{2}$. Its pre-image, under either $h_{i}$, has diameter less than $m / 2 B$. By our construction, both $\operatorname{diam}\left(S_{n k}\right)$, 
$\operatorname{diam}\left(S_{n k}^{\prime}\right)<m / B$, and, hence, both $\operatorname{diam}\left(h_{1} S_{n k}\right), \operatorname{diam}\left(h_{2} S_{n k}^{\prime}\right)<\min \{\epsilon, a / 4\}$. It follows that the rectangle $T=\{(x, y)|| x \mid \leq a / 2,0 \leq y \leq \delta\}$ is covered by cells (in either subdivision) of diameter less than $6 \epsilon$. Each such cell intersects its image under the map, induced by $h, h_{1} h h_{1}^{-1}: T \rightarrow T$, so that points close enough to $p$ are moved an arbitrarily small distance by $h$.

Thus we have that $b$ is a homeomorphism on the complement of the discrete set $P$ of critical points in $M$. Furthermore, for any $p \in P$, there is a sequence $\left\{x_{n}\right\} \subset M-P$ with $x_{n} \rightarrow p$ and $h\left(x_{n}\right) \rightarrow p$. Any such homeomorphism extends by the identity to $P$.

(Necessity). By slightly modifying the argument given above, we may prove: If $S_{1}^{+}, S_{2}^{+}$are two separatrix configurations for the same flow $(M, \phi)$, then there is a self-equivalence of $(M, \phi)$, taking $S_{1}^{+}$onto $S_{2}^{+}$, and the identity on $S_{1}=S_{2}$. It follows that any equivalence $\left(M, \phi_{1}\right) \rightarrow\left(M, \phi_{2}\right)$ induces an equivalence of the associated separatrix configurations.

\section{BIBLIOGRAPHY}

1. N. P. Bhatia and G. P. Szegö, Stability theory of dynamical systems, Die Grundlehren der math. Wissenschaften, Band 161, Springer-Verlag, New York and Berlin, 1970. MR $44 \# 7077$.

2. O. Hájek, Sections of dynamical systems in $E^{2}$, Czechoslovak Math. J. 15 (90) (1965), 205-211. MR 31 \#456.

3. L. Markus, Global structure of ordinary differential equations in the plane, Trans. Amer. Math. Soc. 76 (1954), 127-148. MR 15, 704.

DEPARTMENT OF MATHEMATICS, BOWLING GREEN ST ATE UNIVERSITY, BOWLING GREEN, OHIO 43403 